\title{
Resilience of nuclear matter in light ion induced reactions
}

\author{
M. Colonna, J. Cugnon, ${ }^{*}$ and E. C. Pollacco \\ CEA DAPNIA, CE Saclay, F-91191 Gif-sur-Yvette Cedex, France
}

(Received 23 July 1996)

\begin{abstract}
Cavitation and heating of the target nucleus in the first instances of ${ }^{3} \mathrm{He}$-induced collisions in the $\mathrm{GeV} /$ nucleon range are investigated in an intranuclear cascade model for the formation of this structure and a stochastic one-body dynamics calculation to study its evolution. The hard collisions having essentially ceased when the structure is fully developed, the latter model is particularly suited to study the possible breakup of the system. It is shown, however, that the target recovers a spherical shape rather rapidly, and has thus a good chance to decay by standard evaporation, justifying the use of a cascade + evaporation model to analyze the data. It is also shown that the system has to be much more modified to break up into pieces instead of recovering a compact shape: in these reactions, it is thus expected that nuclear matter is resilient to shape deformation and thermal excitation. Arguments are given to explain that expansion of the system, not important in these reactions, is required to overcome this resilience. [S0556-2813(97)03102-6]
\end{abstract}

PACS number(s): 25.55.-e, 21.65.+f, 24.10.Lx, 25.70.Pq

\section{INTRODUCTION}

Heavy-ion collisions in the incident energy range covering $100 \mathrm{MeV} /$ nucleon to $2 \mathrm{GeV} /$ nucleon are often discussed in terms of a two stage scenario; the first stage corresponds to the formation of thermalized sources and the second one to their decay [1-3]. In general, to simplify the phenomenological investigations, these sources are assumed to acquire a spherical structure. The latter hypothesis is supported by microscopic transport calculations [2]. In accordance, statistical partition [4-7] or evaporative models [8] are applied to describe the evolution from spherical and thermalized emitters. It is, however, understandable that once the dynamical features of the first stage become significant, the thermal energy, angular momentum, shape distortion, and perhaps other effects have to be consistently taken into account in the second stage.

Several theoretical works have recently pointed out that sources with exotic shapes (such as doughnuts, for instance) could be formed in low energy ( $\simeq 20 \mathrm{MeV} /$ nucleon) heavyion collisions [9-11]. However, the conditions under which these shapes could occur in real world are not well known and seem to be so special that, very likely, this should correspond to infrequent events. Formation of transient uneven shapes can very well occur in light ions induced reactions. In Ref. [12], it was suggested that $1 \mathrm{GeV}$ protons drill a conical hole in the target during the first moments of the collisions. Actually, it would be more appropriate to speak of a wake left behind by the incoming proton, since the nuclear density is not vanishingly small in this wake, but is depleted to the $2 / 3$ of its normal value. Antiprotons could give a similar effect, even at very lower energy [13]. Recently, Wang et al. [14] have suggested, in the light of Boltzmann-UehlingUhlenback (BUU) calculations, that this wake effect is amplified by light ions. These predictions depend markedly upon the BUU model. Two models are employed, which

*Permanent address: Physics Department, University of Liege, B-40009 Sart-Tilman Liege 1, Belgium. basically differ by the surface tension. In the first model, with the smaller surface tension, the hole may cover a large fraction of the nuclear volume and last for a relatively long time. In the second model, the hole has a smaller extension and closes, say, $30 \mathrm{fm} / c$ after the beginning of the collision.

The authors of Ref. [14] concentrate on the ${ }^{3} \mathrm{He}+\mathrm{Ag}$ system, for which experimental measurements have been performed in the $0.6-1.8 \mathrm{GeV} /$ nucleon domain [15-18]. Analyses of the measurements have been performed in the frame of the following two-stage model: an intranuclear cascade (INC) model for the formation of the excited source by the hard scattering processes and an evaporation model for its deexcitation (alternatively, an expanding emitting source model [19] has also been used). The INC output (mass, charge, and excitation energy of the residue) is used as input for the evaporation model. It has to be remarked that the junction in time $t_{\text {jun }}$ when to stop the INC and pass on to decay is of consequence. Similar difficulties are encountered with other (BUU, QMD,...) descriptions and relate to the fact that INC does not have all the ingredients to describe the decay. The problem of the determination of $t_{\text {jun }}$ has been carefully investigated in Ref. [20]. In a study of the temporal evolution of a number of parameters (excitation energy, number of ejectiles, and so on) a change in their rates is observed at about the same time. Beyond this time the target phase space density becomes uniform and the system emits nucleons at relatively low energy and with an isotropic distribution. This observation strongly suggests that the $t_{\text {jun }}$ should be identified with this time. This two-stage coupling provides a good description of the bulk of the data $[17,18]$.

The above considerations raise two interesting questions: (i) Does the hole close fast enough for the two-stage model to be applicable (the evaporation model implicitly assuming that the target remnant is spherical at the end of the first stage)? (ii) What are the frequency and characteristics of events in which the target remnant retains an exotic shape when it decays? We address these questions in this paper. Our strategy is the following. We first use the INC model of Refs. [21,22] to investigate the density distribution in the very first moments of the collision. This model using a static 


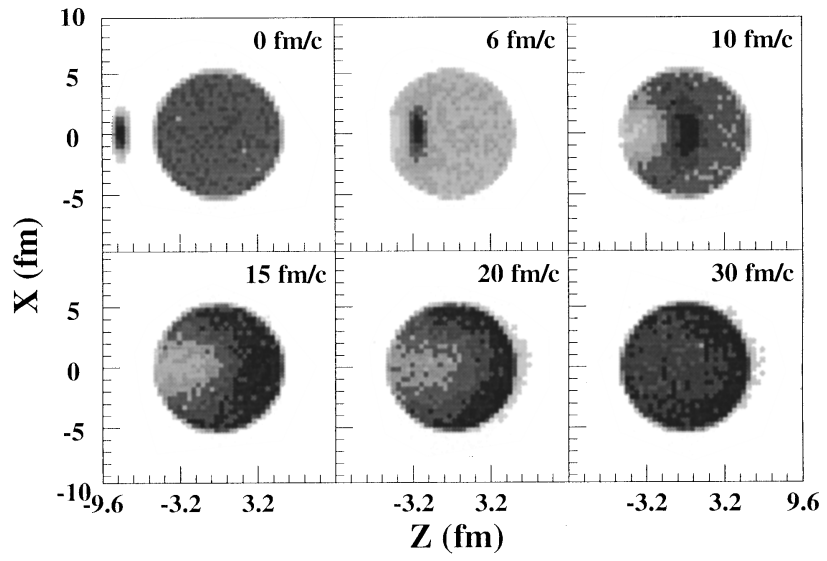

FIG. 1. Nucleon density distribution in the reaction plane for $b=0.1 \mathrm{fm}$ collisions of $1.6 \mathrm{GeV} /$ nucleon ${ }^{3} \mathrm{He}$ projectiles on a $\mathrm{Ag}$ target, at various times, as given by the INC calculation. For $t \lesssim 15 \mathrm{fm} / c$, the nuclear density ahead of the projectile is only slightly modified (the slightly lighter appearance of the target at $t=6 \mathrm{fm} / c$, compared to $t=0 \mathrm{fm} / c$ is an artifact of the plotting routine). The wake left behind by the projectile is clearly visible. The average density at $t=30 \mathrm{fm} / c$ is slightly less than normal nuclear matter density. For sake of clarity, the particles outside the target volume are not displayed.

and constant mean-field potential is not appropriate enough to study the evolution of the shape of the system when the hard baryon-baryon collisions are over and when the hole starts to fill in. To study this stage reliably we employ a stochastic one-body approach (SOBA) [23], which has been proven to be an adequate tool for describing the possible fragmentation of the system coming from the amplification of unstable fluctuations by the mean-field dynamics. Accordingly, the paper is divided as follows. In Sec. II, we present the INC calculations for the ${ }^{3} \mathrm{He}(1.6 \mathrm{GeV} /$ nucleon $)$ $+{ }^{107} \mathrm{Ag}$ collisions. We so determine the shape, the mass, the excitation energy and the (hydrodynamic) velocity field of the target remnant at the time when the hole has the largest extension. In Sec. III, these characteristics are used as input in the SOBA calculation to follow the evolution of the remnant. We extend this analysis to different choices of the input parameters to study under which circumstances exotic shapes would fragment before reforming a spherical remnant and to look for a possible characterization of such fragmentation. In Sec. IV, we shortly discuss the validity of the two-stage picture for light ion induced collisions. Finally, Sec. V contains our conclusion.

\section{CAVITATION IN THE FIRST STAGE OF THE COLLISIONS}

In order to investigate the deformation of the target density distribution during the first moments of the collision, we performed an INC calculation of the collisions of 1.6 $\mathrm{GeV} /$ nucleon ${ }^{3} \mathrm{He}$ projectiles on ${ }^{107} \mathrm{Ag}$ targets. The detail of the INC model can be found in Refs. [21,22]. The average density profile in the reaction plane for $b=0.1 \mathrm{fm}$ collisions is given in Fig. 1 and was obtained by considering 4000 runs. The wake left behind by the projectile is clearly visible and is mainly developed for $t \simeq 15-20 \mathrm{fm} / c$. At this time, the density depression reaches $\rho_{0} / 2, \rho_{0}$ being normal nuclear

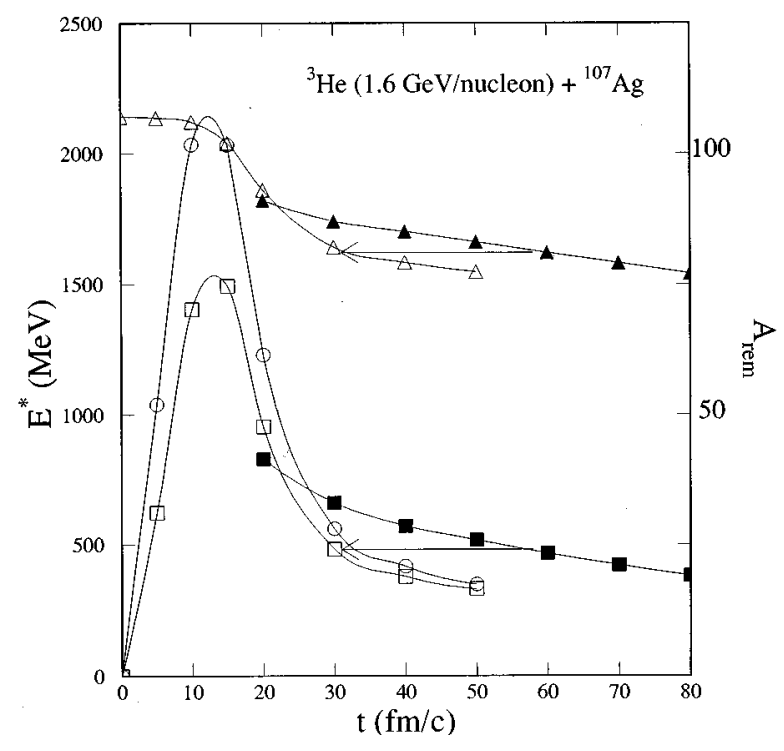

FIG. 2. Time evolution of the excitation energy $E^{*}$ (lower curves, scale on the left) and of the target mass number $A_{\text {rem }}$ (upper curves with triangles, scale on the right) in $b=0.1 \mathrm{fm}$ collisions of $1.6 \mathrm{GeV} /$ nucleon ${ }^{3} \mathrm{He}$ ions on a $\mathrm{Ag}$ target. The open symbols correspond to the INC calculation and the black symbols to the SOBA calculation using input data extracted from the INC results at $t \sim 20 \mathrm{fm} / c$. The curve with the open squares is obtained from the curve with the open circles by removing the mass energy of the $\Delta$ particles. See text for more detail.

matter density. Incidentally, we want to point out that we used the experimental density distribution for ${ }^{3} \mathrm{He}$ (see Refs. $[24,25]$ for details). The more important wake predicted in the first calculation of Ref. [14], that is called cavitation, may be due to the choice of a too compact configuration for this projectile. In any case, the formation of a wake in both BUU and INC calculations is due to the initial nucleon-nucleon collisions. The mean field plays a minor role in the formation process. In our calculation, the wake disappears progressively after $15-20 \mathrm{fm} / c$ because nucleons are running into the partially empty space. At $30 \mathrm{fm} / c$, the density is almost uniform (this corresponds to the time $t_{\text {jun }}$ at which the rate of variation of the number of ejectiles and of the excitation energy is changing slope, as we indicated in the Introduction). This development is favored by the presence, in the INC model, of a constant potential well in the target volume. Nucleons traveling backwards can be reflected by the potential wall. It is expected that, in reality the potential well, or more exactly the mean field, is deformed according to the density distribution although it is not clear that it follows instantaneously the density distribution, when the latter is perturbed by very hard collisions, since the mean-field dynamics is basically associated with soft processes. In the next section, the evolution of the wake is studied by incorporating the dynamics of the mean field.

The time evolution of the target excitation energy $E^{*}$ and of the target remnant mass $A_{\text {rem }}$, defined as the excitation energy and mass number inside the original target volume [12], is displayed in Fig. 2. As noted in the Introduction, there is clearly a change in the corresponding curves around $t \sim 30 \mathrm{fm} / c$, which can be identified as $t_{\text {jun }}$. We will come back to this figure in Sec. IV. 
To investigate the average (or hydrodynamical) current inside the target, we divide the space in cells and compute the following quantity

$$
\vec{j}(\vec{r})=\left\langle\frac{1}{\Delta v} \sum_{i \in \Delta v} \frac{\vec{p}_{i}}{m_{i}}\right\rangle,
$$

where $\vec{r}$ is the position of the center of the cell, $\Delta v$ is the volume of a cell and $\vec{p}_{i}$ is the momentum of nucleon $i$. The brackets indicate the average over the runs. The velocity field is given by

$$
\vec{v}(\vec{r})=\frac{\vec{j}(\vec{r})}{\rho(\vec{r})}
$$

The linear size of the cells is about $0.6 \mathrm{fm}$. We discuss the main properties of the velocity field. At the beginning of the collision, the velocity field is concentrated on the projectile. As time evolves, the longitudinal current spreads considerably, reflecting the sideward diffusion of the particles involved in the current (see Fig. 1). It is also largely slowing down. This does not mean, however, that there are no fast particles. But the latter are contributing only slightly to the macroscopic current. As expected, the velocity field acquires a perpendicular component. This current is much smaller than that along the $z$ direction, even when the wake is fully developed. Just to give an idea, let us mention that, at that time, the $z$ component of the velocity field is maximum a little bit ahead of the apex of the cone, with a value of $\beta \sim 0.2$. The perpendicular component has a maximum absolute value of $\sim 0.07$.

The formation of a wake is observed for impact parameters up to $3 \mathrm{fm}$, with an amplitude that decreases with increasing impact parameter. For all higher impact parameters, the density is only slightly modified, and the nucleus essentially retains a spherical shape. Therefore, we can safely put an upper limit on the "cross section for the wake formation" of the order of $270 \mathrm{mb}$ for this system. The total cross section being of the order of 2 barns, the fraction of this kind of events is of the order of 10 to $15 \%$ at the most. This fraction is expected to be the same for medium heavy and heavy targets.

\section{RESILIENCE TO SHAPE DEFORMATION AND THERMAL EXCITATION}

\section{A. Cascade shapes}

As noted in the previous section, INC calculations are not adapted to describe the nuclear shape once the hard collisions have ceased and dynamical mean-field effects become important. To study this question, we consider a SOBA calculation with input corresponding to the time when the wake is the mostly developed, i.e., around $15-20 \mathrm{fm} / c$ in the case of Fig. 1. The detail of the calculation can be found in Ref. [23]. It is sufficient to say that this transport model uses the test particle method (here, we use typically 50 test particles per nucleon) and that a noise is introduced at the beginning of the calculation. The noise is suitably tuned in order to account for the thermal fluctuations associated with the random nature of nucleon-nucleon collisions, as described by

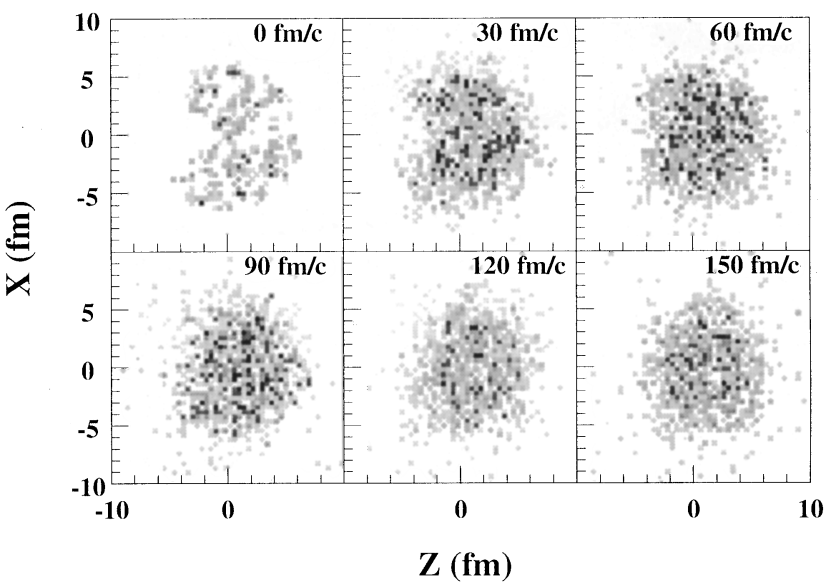

FIG. 3. Nucleon density distribution in the reaction plane at various times, as given by the SOBA calculation for a configuration corresponding to a $\mathrm{Ag}$ target at $T=8 \mathrm{MeV}$ after removal of a cone. The Gaussian parameter $\sigma$ is taken as $0.9 \mathrm{fm}$. The system contains 91 nucleons. The density scale is arbitrary, but the darkest zones correspond to the largest values of the density.

the Boltzmann-Langevin theory [26]. This method has been shown to be accurate for describing the dynamics of the fluctuations of the phase space distribution function, especially when the system enters the so-called spinodal region [27]. More precisely, in this Ref. [27], it is explicitly shown that this method gives similar results for the spinodal decomposition, as the two-dimensional complete BoltzmannLangevin calculation on a lattice.

Furthermore, it has been shown that the treatment of the mean field requires some special care. For simple Skyrmelike effective potentials, the mean field is a function of the local density and the latter is calculated by providing the test particles with a Gaussian of width parameter $\sigma$. As shown in Ref. [28], in semiclassical calculations, a value of $\sigma=0.9 \mathrm{fm}$ is necessary to provide a good approximation to the quantal description of spinodal instabilities. Since we are primarily interested in the bulk instabilities of the remnant, we chose this value. We stress the fact that a smaller value of this parameter as $\sigma=0.5 \mathrm{fm}$ is recommended to get a good description of the nuclear surface in the ground state with a classical test particle method, but it is not suitable for tracking the possible bulk instabilities.

In order to simplify the transition between the two calculations, we idealized the state of the system as predicted by the INC. To start, we construct a Ag nucleus, from which a cone has been removed, with the apex at the center of the nucleus and with opening angle similar to the one of the wake observed in Fig. 1. The system is initialized at temperature $T=8 \mathrm{MeV}$. This corresponds to an excitation energy slightly less than the thermal excitation energy predicted by the INC calculation. The spatial distribution of the test particles is disturbed randomly (noise) in concordance with the known density fluctuations of a Fermi gas at this temperature. The evolution of the density, in a representative run, is given by Fig. 3. It can be seen that, in less than 60 $\mathrm{fm} / c$ the system loses a number of test particles and rapidly forms a compact spherical shape. Our results show that nuclear matter is resilient to the deformation and heating produced in the INC stage, inasmuch as, by comparing this 


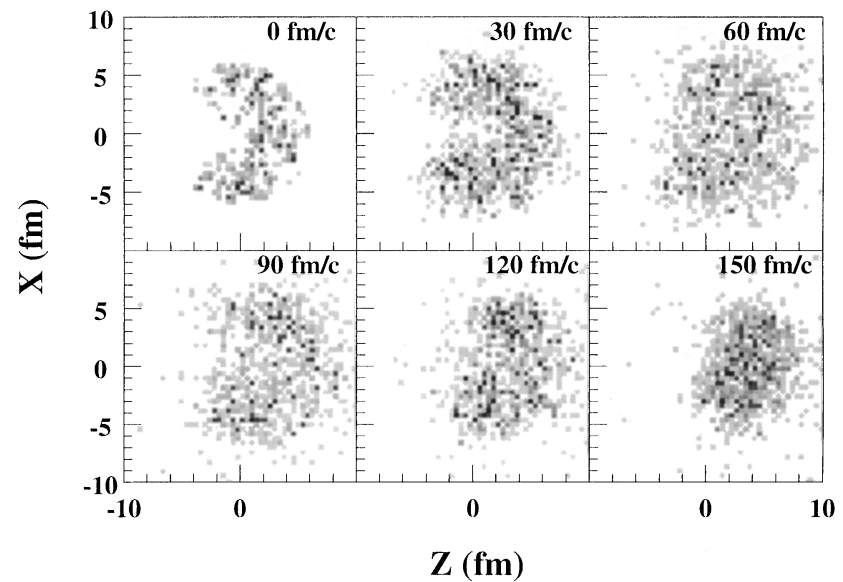

FIG. 4. Same as Fig. 3, with $T=6 \mathrm{MeV}$ and the velocity field extracted from the INC calculation. See text for detail.

figure and Fig. 1, one can realize that the idealization of the starting configuration used in the SOBA calculation is certainly less favorable for shape resilience, since the density inside the cone is not vanishing in the INC calculation.

In addition, we also performed a SOBA calculation by imposing the velocity field, as given by the INC calculation at $t \simeq 15 \mathrm{fm} / c$. For this case, we used $T=6 \mathrm{MeV}$, which in principle is more favorable to the development of spinodal instabilities [29]. The resilience of the nuclear system holds for this case also, as shown by Fig. 4, where a typical event is displayed. Compared to the previous case, the shape is more perturbed at the beginning, because of the velocity field, but the spherical symmetry is also basically recovered within $t \sim 60 \mathrm{fm} / c$. It is, however, interesting to note that the system size grows much more than in the previous case (Fig. 3): at $T=8 \mathrm{MeV}$, the system cools by an important emission of energetic particles leaving behind a "core" moderately excited and rather stable; at $T=6 \mathrm{MeV}$, this emission is considerably diminished since the energetic particles leave the system less easily, thus developing, apparently, more efficiently a macroscopic pressure. The ensuing expansion, triggered by the latter creates larger fluctuations in the volume of the system, leading to more instability. This motivates our remark above about the choice of the temperature. However, in the present case, this effect is not strong enough to allow the breaking of the system.

For the sake of comparison, we have considered SOBA calculations with the parameter $\sigma=0.5 \mathrm{fm}$. The resilience is then less apparent. For $T=6 \mathrm{MeV}$ and the velocity field the system recovers sphericity, somewhat slower, in $85 \%$ of the 40 events that we calculated. It breaks down, mainly in two pieces, in the remaining events. For $T=8 \mathrm{MeV}$ and the velocity field the system recovers sphericity in $2 / 3$ of the 25 calculated events. We think this higher proportion comes from the fact that at $T=8 \mathrm{MeV}$, with a small surface tension, the system loses test particles at a higher rate, approaching vaporization.

\section{B. Exotic shapes}

In view of the results obtained so far, we addressed the following questions: To what degree should the system be distorted in order to break down into pieces instead of going

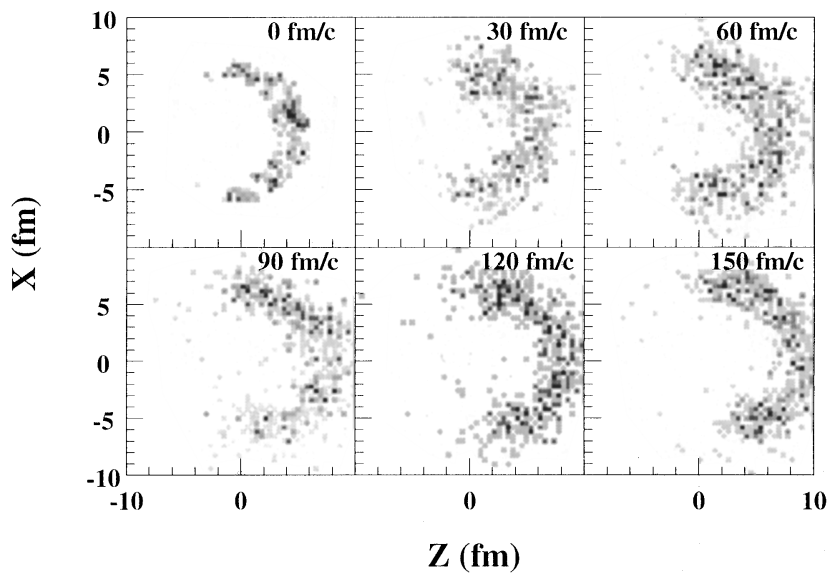

FIG. 5. Same as Fig. 4, but for an initial configuration corresponding to an excavated Ag nucleus. The maximum thickness in the longitudinal $(z)$ direction is equal to half of the original radius. The system contains 70 nucleons. See text for detail.

back to the spherical shape? Is the shape (that we suspect to be) realized in ${ }^{3} \mathrm{He}$ induced reactions far from the onset of this multifragmentation process? These questions are very hard to answer as there are many ways of distorting the system. We restrict our study only to one of the possible extrapolations of the distortion realized in the INC, but we think it is already meaningful. We considered a much more excavated configuration, with only one quarter of the diameter along the incident direction remaining in the target (see Fig. 5). The so-prepared remnant contains 70 nucleons at a temperature of $6 \mathrm{MeV}$. Furthermore, the velocity field discussed in the previous subsection is superimposed. As this shape is characterized by a large surface-to-volume ratio, it is expected that the bulk instabilities give way, at least partially, to the surface instabilities. Therefore, taking the Gaussian parameter as $\sigma=0.5 \mathrm{fm}$ is expected to reveal the latter more easily. But, for the sake of comparison, we also performed SOBA calculations for this starting configuration with $\sigma=0.9 \mathrm{fm}$. The results for a typical event are illustrated in Fig. 5. They are rather surprising as the system remains connected (a single part) for a rather long time, although it is not really going rapidly to a spherical shape. A closer inspection of the results seems to indicate that the relaxation of the system is very long because there is a near cancellation between the Coulomb forces, which tend to deplete the system along the collision axis, leading to an almost annular shape, and the surface tension, which tends to make the shape more compact. The velocity field works against the surface tension, as the Coulomb forces. The results are radically different in a SOBA calculation, with this time $\sigma=0.5 \mathrm{fm}$. In this case (see Fig. 6) the system breaks down into pieces rather rapidly. In this typical example, the system breaks first in a piece traveling forward and a not complete annular ring which breaks into two pieces that are seen in Fig. 6 by their cross section in the reaction plane. Once again the velocity field is instrumental in this breaking. It is interesting to look at the mass distribution of the fragments, in the case of the last calculation. It is given in Fig. 7 (for 50 events) and shows that the mean mass of the fragments is of the order of 12. This is significantly smaller than the average mass of the fragments coming from a spinodal decomposition (about 20- 


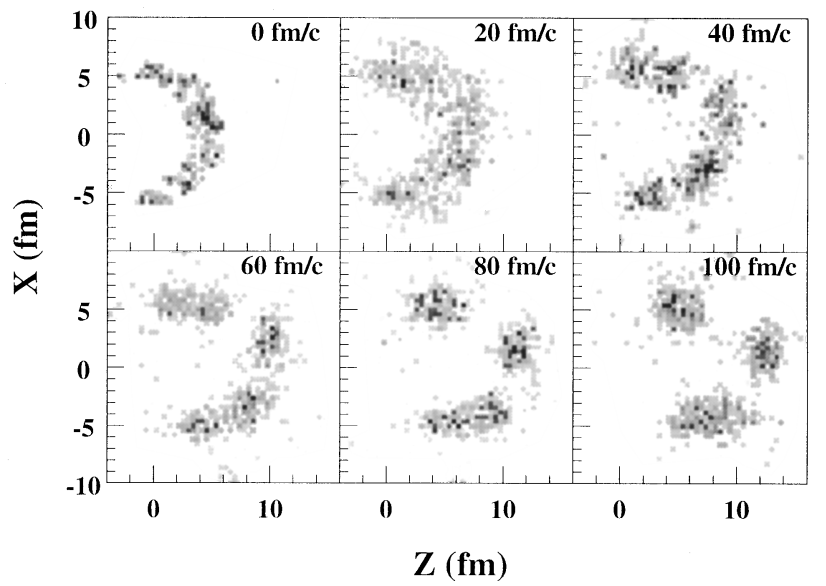

FIG. 6. Same as Fig. 5 for $\sigma=0.5 \mathrm{fm}$.

30), as predicted in Refs. [23,29]. This observation, which alone can by no means be taken as a clear signature, is nevertheless consistent with an interpretation of the fragmentation observed in this calculation as due to a surface instability $[10,30]$. The role of the Coulomb force (between the ends of the system) and of the velocity field need to be investigated carefully before drawing a definite conclusion.

\section{VALIDITY OF THE TWO-STEP PICTURE}

The considerations of Sec. III A indicate that the system basically recovers a spherical shape after a relatively short time $(\$ 60 \mathrm{fm} / c)$, before continuing to deexcite presumably by standard evaporative processes (or by the emission of intermediate mass fragments when the remaining excitation energy is large enough). It is interesting at this point to make a comparison between the (average) evolution of the system in INC and SOBA. This is provided by Fig. 2. The evolution of the thermal energy (the mass energy of the $\Delta$ particles, not introduced in the SOBA calculation, has been subtracted to facilitate the comparison) and of the remnant mass are given
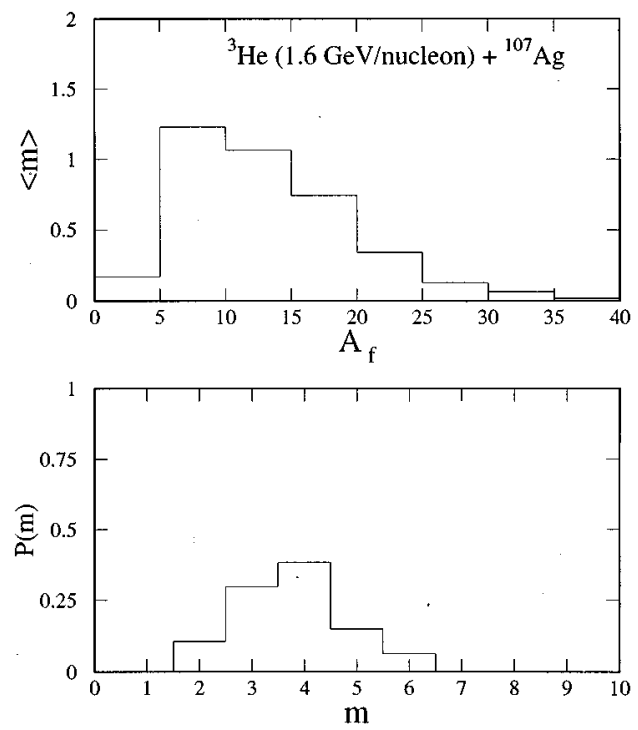

FIG. 7. Average number $m$ of fragments of mass $A_{f}$ (upper part) and probability distribution for having $m$ fragments (lower part), in the SOBA calculation of Fig. 6. by open symbols for INC and black symbols for SOBA. There is a remarkable similarity between the two calculations when the time scale for SOBA is compressed by a factor three relative to the INC time scale. A possible explanation lies in the fact that, although the matter and momentum distributions in the INC are fairly homogeneous at $t \simeq 15-20 \mathrm{fm} / c$, the target has still small packets of fast particles, which, by escaping on a short time scale, rapidly cool the system. In SOBA, smoothing gives rise to a slower energy depletion. More importantly, there is a good correspondence (translated by the arrows in Fig. 2) between the values of the excitation energy and the mass of the remnant at the time the system is spherical again in the INC+SOBA calculation $(t \simeq 60 \mathrm{fm} / c)$ on the one hand, and the values of the same quantities in the INC calculation alone at the time $(t \simeq 30 \mathrm{fm} / c)$ when the system is spherical and more or less homogeneous, on the other hand. These considerations justify to some degree the use of a cascade + evaporation (or the like) model, with the choice of the stopping time recommended in Ref. [20], to analyze the data. Note that the agreement between the properties of the spherical configurations in the two calculations shown in Fig. 2 is obtained with extreme conditions, namely a maximum development of the wake and an idealization of the hole in SOBA calculation.

\section{DISCUSSION}

In this paper, we investigate the appropriateness of the cascade + deexcitation model for analyzing the experimental data relative to light ion induced reactions in the $\mathrm{GeV} /$ nucleon range. A necessary condition is that the system is thermalized and basically spherical when the hard collision phase (cascade) comes to an end. We have seen that this is the case for the ${ }^{3} \mathrm{He}+\mathrm{Ag}$ system at $1.6 \mathrm{GeV} /$ nucleon incident energy. Consequently, we consider that this should also be the case for lower energy and smaller projectiles up to at least the same energy. Note that the wake predicted for $2 \mathrm{GeV}$ protons corresponds to a density depleted of only $2 / 3 \rho_{0}$ and reaches its full development at $8 \mathrm{fm} / c$ [12]. For these systems, the possibility of having a fragmentation before the evaporation or an evaporation from an exotic shape remnant is very unlikely. Taking into account the results of Sec. III A for the ${ }^{3} \mathrm{He}+\mathrm{Ag}$ system, we can put a very conservative upper bound of $\sim 90 \mathrm{mb}$ on the cross section for the occurrence of these special events, taking into account the least favorable (for resilience) of our results and the fact that the wake is of decreasing importance as the impact parameter is increasing. Our results with the proper choice $\sigma=0.9 \mathrm{fm}$ show that the cross section for these special events is, very likely, vanishingly small. In any case, our investigations indicate that nuclear matter is particularly resilient to deformation and to thermal excitation in this kind of reactions. Note that in central collisions, the excitation energy of the remnant after the cascade phase could be as high as $600 \mathrm{MeV}$, i.e., $\sim 7 \mathrm{MeV} /$ nucleon, as indicated in Fig. 2 and confirmed by the experimental data [17]. The situation is rather contrasted with the one prevailing in central heavy ion collisions [31], where multifragmentation occurs frequently for a similar estimated excitation energy per nucleon. Although it is not yet clear whether the emission of several intermediate mass fragments is a rapid process or simply a 
slow evaporative process, there are good indications [32] that the system is then less resilient to a rapid breakup in intermediate mass pieces. A possible explanation may rest on the fact that the system is not significantly compressed in light ion reactions. On the other hand, in heavy ion collisions the system is presumably more compressed, leading to a collective expansion. This in turn will lead to volume instabilities which facilitate the the breakup of the system [23]. This issue deserves extended investigations.

Coming back to the ${ }^{3} \mathrm{He}+\mathrm{Ag}$ system, the possibility of having deformed shapes leading to unusual decay patterns is certainly increasing with energy. One may speculate on the formation of very excavated shapes such as the one we considered in Figs. 5 and 6. They may provide an appealing opportunity to investigate possible surface instabilities [30] of nuclear matter. This point also deserves further analyses.
[1] J. Gosset et al., Phys. Rev. C 16, 629 (1977).

[2] J. Cugnon and S. E. Koonin, Nucl. Phys. A355, 477 (1981).

[3] N. Marie et al., Phys. Lett. (to be published).

[4] D H. E. Gross, Rep. Prog. Phys. 53, 605 (1990).

[5] J. Bondorf et al., Nucl. Phys. A443, 321 (1985).

[6] H. Stöcker and W. Greiner, Phys. Rep. C5, 277 (1986).

[7] J. Konopka et al., Phys. Rev. C 50, 2085 (1994).

[8] L. G. Moretto, Prog. Part. Nucl. Phys. 21, 401 (1988).

[9] W. Bauer et al., Phys. Rev. Lett. 69, 1888 (1992).

[10] L. G. Moretto et al., Phys. Rev. Lett. 69, 1884 (1992).

[11] B. Borderie et al., Phys. Lett. B 302, 15 (1993).

[12] J. Cugnon, Nucl. Phys. A462, 721 (1987).

[13] P. Jasselette, J. Cugnon, and J. Vandermeulen, Nucl. Phys. A484, 542 (1988).

[14] G. Wang, K. Kwiatkowski, V. E. Viola, W. Bauer, and P. Danielewicz, Phys. Rev. C 53, 1811 (1996).

[15] K. Kwiatkowski et al., Phys. Rev. C 49, 1516 (1994).

[16] K. B. Morley et al., Phys. Lett. B 355, 52 (1995).

[17] E. C. Pollacco et al., in Advances in Nuclear Dynamics, Proceedings of the 12th Winter Workshop on Nuclear Dynamics, Snowbird (1996), edited by W. Bauer and G.D. Westfall (to be published).

[18] L. Pienkowski et al., Phys. Lett. B 336, 147 (1994).

[19] W. A. Friedman, Phys. Rev. C 42, 667 (1990) .
[20] L. Pienkowski et al., Proceedings of the Winter Meeting on Nuclear Physics, Bormio, 1993, edited by I. Iori (RSEP, Milano, 1993).

[21] J. Cugnon, Nucl. Phys. A389, 191c (1982).

[22] J. Cugnon and M. -C. Lemaire, Nucl. Phys. A489, 781 (1988).

[23] A. Guarnera, M. Colonna, and Ph. Chomaz, Phys. Lett. B 373, 267 (1996).

[24] C. Ciofi degli Atti, Nucl. Phys. A543, 183c (1992).

[25] R. Schiavilla, V. R. Pandharipande, and W. R. Wiringa, Nucl. Phys. A449, 219 (1986).

[26] S. Ayik and C. Grégoire, Nucl. Phys. A513, 187 (1990).

[27] G. F. Burgio, Ph. Chomaz, M. Colonna, and J. Randrup, Nucl. Phys. A581, 356 (1995).

[28] S. Ayik, M. Colonna, and Ph. Chomaz, Phys. Lett. B 353, 417 (1995).

[29] Ph. Chomaz, M. Colonna, A. Guarnera, and B. Jacquot, Nucl. Phys. A583, 305c (1995).

[30] L. G. Moretto and G. J. Wozniak, Annu. Rev. Nucl. Sci. 43, 379 (1993).

[31] Y. G. Ma et al., Surveying the Caloric Curve, LPC Caen preprint, 1996 (to be published).

[32] D. Durand et al., LPC Caen Report No. LPCC-15, 1995 (to be published). 